\title{
CONGENITAL HEART DISEASE IN FETAL AND PEDIATRIC AUTOPSIES: A 15-YEAR RETROSPECTIVE REVIEW AT A SINGLE TEACHING HOSPITAL IN BANGKOK, THAILAND
}

\section{Mana Taweevisit ${ }^{1}$, Paul Scott Thorner ${ }^{1,2}$}

\section{Department of Pathology, Faculty of Medicine, Chulalongkorn University, Bangkok, Thailand \\ 2Department of Laboratory Medicine and Pathobiology, University of Toronto, Toronto, Canada}

Background and aims: Congenital heart defects carry significant morbidity and mortality in the pediatric age group. This study determined the spectrum of congenital heart defects in our fetal and pediatric autopsy population, and the association with extra-cardiac malformations and chromosome abnormalities.

Methods: Autopsy reports over a 15-year period were reviewed for cases with congenital heart defects. Post-mortem findings were correlated with echocardiography records when available.

Results: Of 608 autopsies, 119 (19\%) had congenital heart defects, with the greatest proportion in the neonatal group (53\%). Persistent left superior vena cava and hypoplastic left heart syndrome were the most common defects. Extra-cardiac malformations were present in $41 \%$ of cases, the most common of which were dysmorphic features. One case each of CHARGE association and Meckel-Gruber syndrome were identified. Chromosome abnormalities were present in $18.5 \%$ of cases, the most common of which was trisomy 18 . All cases had been identified pre-mortem. Prenatal echocardiography was available in 52 cases and there was $85 \%$ correlation between echocardiogram and cardiac autopsy findings. Defects missed by echocardiography were generally of mild severity. One case interpreted as hypoplastic left heart syndrome turned out to be double outlet right ventricle. Discordances between echocardiogram and autopsy cardiac findings did not impact patient management.

Conclusions: Autopsy examination is important to delineate the anatomy of cardiac defects as well as recognize extra-cardiac malformations for identification of possible genetic syndromes. This information can then be used for parental counselling as well as for internal assessment of the accuracy of pre-mortem imaging studies

\section{References:}

1. Bernier PL, Stefanescu A, Samoukovic G, Tchervenkov Cl. The challenge of congenital heart disease worldwide: epidemiologic and demographic facts. Semin Thorac Cardiovasc Surg Pediatr Card Surg Annu. 2010;13:26-34.

2. Gilbert-Barness E, Debich-Spicer D. Congenital malformations of the heart. In: Gilbert-Barness E (ed.) Potter's Pathology of the Fetus, Infant and Child. Philadelphia PA: Mosby Elsevier; 2007: pp. 987-1024.

\begin{tabular}{|l|c|c|c|c|}
\hline Category & Total & $\begin{array}{c}\text { Found in } \\
\text { isolated }\end{array}$ & $\begin{array}{c}\text { As a part of } \\
\text { complex }\end{array}$ & P \\
\hline Septal defects & 38 & 18 & 20 & 0.74 \\
\hline Atrial septal defect & 7 & 6 & 1 & \\
\hline Ventricular septal defect & 13 & 6 & 9 & \\
\hline Atrioventricular septal defect & 8 & 4 & 6 & \\
\hline Patent ductus arteriosus & 6 & 2 & 4 & \\
\hline Left ventricular outflow tract obstruction & 36 & 14 & 22 & 0.23 \\
\hline Hypoplastic left heart syndrome & 27 & 8 & 19 & \\
\hline Interrupted aortic arch & 1 & 1 & - & \\
\hline Coarctation of aorta & 5 & 4 & 1 & \\
\hline Bicuspid aortic valve & 3 & 1 & 2 & \\
\hline Right ventricular outflow tract obstruction & 24 & 14 & 10 & 0.41 \\
\hline Pulmonary stenosis & 3 & 1 & 2 & \\
\hline Bicuspid pulmonic valve & 4 & 2 & 2 & \\
\hline Ebstein anomaly & 11 & 10 & 1 & \\
\hline Triscuspid stenosis & 1 & 1 & - & \\
\hline Pulmonic atresia & 4 & - & 4 & \\
\hline Tricuspid atresia & 1 & - & 1 & \\
\hline Anomalous great vessels & 34 & 8 & 26 & 0.003 \\
\hline Persistent left superior vena cava & 26 & 4 & 22 & \\
\hline Vascular ring & 2 & 2 & - & \\
\hline Major aortopulmonary collateral arteries & 4 & 1 & 3 & \\
\hline Varices & 1 & 1 & - & \\
\hline Right-sided aortic arch & 1 & - & 1 & \\
\hline Conotruncal anomaly & 20 & 10 & 10 & 1.00 \\
\hline Truncus arteriosus & 11 & 6 & 5 & \\
\hline Tetralogy of Fallot & 2 & 1 & 1 & \\
\hline Double outlet of right ventricle & 7 & 3 & 4 & \\
\hline Connection anomaly & 13 & 3 & 10 & 0.51 \\
\hline Transposition of great arteries & 7 & 3 & 4 & \\
\hline Anomalous pulmonary venous return & 6 & - & 6 & \\
\hline Other anomalies & 11 & 5 & 6 & \\
\hline Endocardial fibroelastosis & 7 & 3 & 4 & \\
\hline Absence of ductus arteriosus & 1 & 1 & - & \\
\hline Ectopia cordis & 1 & 1 & - & \\
\hline Cor triatriatum & 1 & - & 1 & \\
\hline Dextrocardia & 1 & - & 1 & \\
\hline Total & 176 & 72 & 104 & 0.03 \\
\hline & & & & \\
\hline
\end{tabular}

\begin{tabular}{|c|c|c|c|c|c|c|c|c|c|c|c|}
\hline Category & $\begin{array}{l}\frac{\vec{\pi}}{0} \\
\stackrel{0}{10}\end{array}$ & 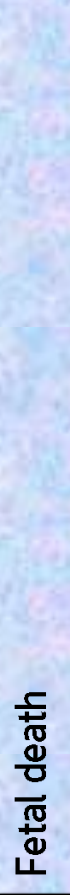 & 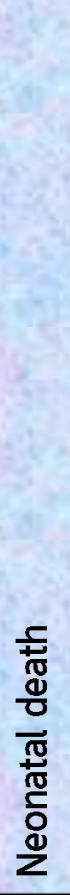 & 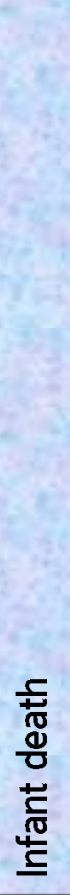 & 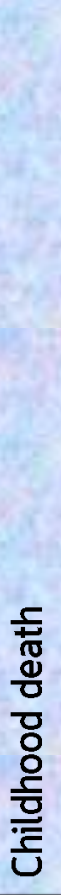 & 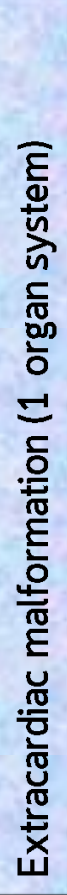 & 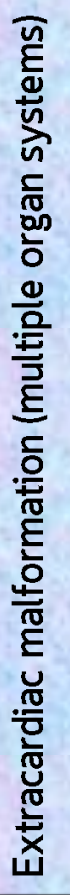 & 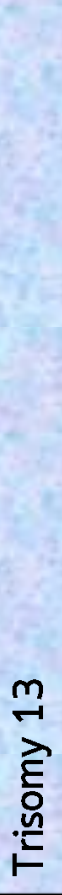 & 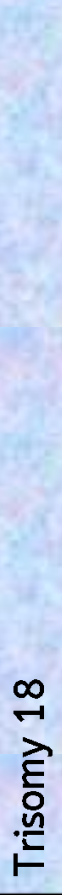 & 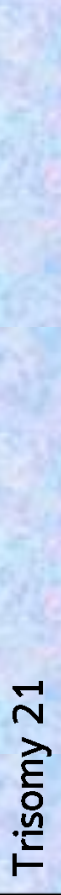 & 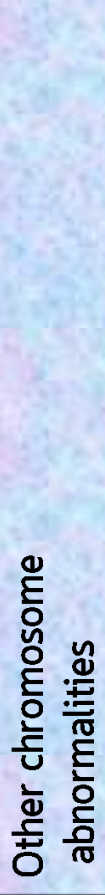 \\
\hline Septal defects & 18 & 6 & 6 & 5 & 1 & 3 & 8 & 2 & 1 & 3 & 0 \\
\hline LVOTO & 14 & 3 & 7 & 4 & 0 & 1 & 4 & 0 & 2 & 1 & 2 \\
\hline RVOTO & 14 & 6 & 7 & 0 & 1 & 0 & 1 & 0 & 0 & 1 & 0 \\
\hline Anomalous vessels & 8 & 2 & 5 & 1 & 0 & 0 & 6 & 1 & 2 & 0 & 0 \\
\hline Conotruncal anomaly & 10 & 1 & 7 & 2 & 0 & 2 & 3 & 0 & 1 & 0 & 0 \\
\hline Other anomalies & 5 & 3 & 1 & 1 & 0 & 0 & 0 & 0 & 0 & 0 & 0 \\
\hline Total & 72 & 21 & 35 & 14 & 2 & 6 & 23 & 3 & 6 & 4 & 2 \\
\hline
\end{tabular}

LVOTO = Left ventricular outflow tract obstruction; RVOTO = Right ventricular outflow obstruction 